\title{
Local Organising Committee
}
M. Towrie
Rutherford Appleton Laboratory
P. Matousek
Rutherford Appleton Laboratory
S. M. Tavender
Rutherford Appleton Laboratory
M. W. George
University of Nottingham
J. N. Moore
University of York
$\mathrm{W}$. T. Toner
Clarendon Laboratory
J. J. Turner
University of Nottingham
D. Bolsom
G. Gaborel
R. E. Hester
Rutherford Appleton Laboratory
Rutherford Appleton Laboratory
University of York
A. W. Parker
Rutherford Appleton Laboratory

Conference Liaison Office

(Rutherford Appleton Laboratory)

S. C. Hilton

J. A. Graham

P. D. Athawes

M. E. Poster

International Organising Committee:
A. C. Albrecht, USA
S. Mukamel, USA
G. H. Atkinson, USA
A. B. Myers, USA
M. El-Sayed, USA
A. W. Parker, UK
Ch. Flytzanis, france
F. Siebert, Germany
H. Hamaguchi, Japan
T. G. Spiro, USA
R. E. Hester, UK
M. Stockburger, Germany
R. M. Hochstrasser, USA
H. Takahashi, Japan
C. Kitagawa, Japan
M. Tasumi, Japan
T. Kobayashi, Japan
H. P. Trommsdorff, France
J. A. Koningstein, Canada
D. Wiersma, The Netherlands
Y. Koyama, Japan
R. Wilbrandt, Denmark
A. Lau, Germany
W. H. Woodruff, USA
A. Laubereau, Germany
L. D. Ziegler, USA
J. L. Martin, France 